\title{
Change detection support for supervisory controllers of highly automated systems: Effects on performance, mental workload, and recovery of situation awareness following interruptions
}

\author{
Rick van der Kleij ${ }^{\text {a, }}$, Tom Hueting ${ }^{\text {a }}$, Jan Maarten Schraagen ${ }^{\text {a, b }}$ \\ ${ }^{a}$ Human Behaviour \& Organisational Innovations, Netherlands Organisation for Applied Scientific Research (TNO), The Netherlands \\ ${ }^{\mathrm{b}}$ Department Cognitive Psychology and Ergonomics, University of Twente, The Netherlands
}

\section{A R T I C L E I N F O}

\section{Article history:}

Received 10 January 2017

Received in revised form

24 October 2017

Accepted 12 February 2018

\section{Keywords:}

Situation Awareness Recovery (SAR)

Dynamic positioning

Management-by-exception

Out-of-the-loop (OOTL) performance problem

Human-automation collaboration

\begin{abstract}
A B S T R A C T
Dynamic Positioning (DP) is a computer-controlled process to automatically keep a floating vessel at a specific position or to follow a pre-defined path (tracking) by using its own propellers and thrusters. The human supervisory controller has no direct need to constantly know what the status is of all parts of the automation and the system it is controlling, because the highly automated DPS is controlling all components itself. Only after a failure arises, the operator needs to take over manual control and take appropriate action(s) to prevent the failure from harming the operation. As the supervisory controller may be out of the loop, swiftly taking over control may be problematic when failures arise. The purpose of the current study was to investigate whether automation of change detection enables human operators with low awareness of the automation and the system it is controlling to quickly recover awareness in emergency take-over situations. A 2 by 2 within subjects experiment was conducted using a DP simulation $(\mathrm{n}=22)$. Within-subjects factors were support (Yes, No) and interruption (Yes, No). Results showed that change detection support helps in the process of recovering situation awareness after it has been reduced, due to an interruption of the primary task of overseeing the automation. Interestingly, support was not beneficial to the participants in all conditions. In non-interrupted conditions the support unexpectedly resulted in higher workload, raising questions whether supervisory controllers should be supported continuously or only when it is required. Relevance to industry: The results show that change detection support has potential value in operational maritime environments, especially in situations where the DP operator has low situation awareness. Future research should investigate whether adaptive aiding could alleviate some of the negative effects of non-adaptive operator support in maritime environments.
\end{abstract}

๑ 2018 Published by Elsevier B.V.

\section{Introduction}

Many systems operate autonomously or are envisioned to do so in the near future. Such systems have the capacity to operate independently without human participation in the task execution phase (this does not preclude human involvement during other stages). An autonomous system may function fine for the range of situations it is designed to address. More interesting is what happens in situations that were not foreseen by its makers (cf. Bainbridge, 1983; Woods and Cook, 2006). Boundaries are reached

\footnotetext{
* Corresponding author. TNO, Kampweg 5, P. O. Box 23, 3769 ZG Soesterberg, The Netherlands.

E-mail address: Rick.vanderKleij@tno.nl (R. van der Kleij).
}

when situations become manifest that the system designers have not anticipated or foreseen. For instance, when a critical system component fails, when critical parameters are exceeded, or an unforeseen incident occurs, an anomaly response is required, usually from a human operator. Hence, autonomous systems need a human operator as backup, in case the automation fails, and the ability is required to improvise and use flexible procedures (cf. Fitts, 1951). It is therefore better not to use the term 'autonomous', but to refer to these systems as semi-autonomous, reflecting the active involvement of a human operator as backup. Semi-autonomous systems, which can be characterized by varying degrees of autonomous capabilities, will retain the possibility of manual control and may require an engaged human operator to monitor the system and assume control under conditions when the system cannot control 
itself (Rousseau and Crane, 2016).

A special instance of a semi-autonomous system is a Dynamic Positioning System, or DPS in short. A DPS is a computer-controlled system to automatically keep a floating vessel at a specific position or to follow a pre-defined path (tracking) by using its own propellers and thrusters (Fossen, 1994). Applications include shuttle tanker operations, deep water drilling (e.g., drilling rigs), dredging and rock dumping, pipe laying and pipe trenching operations, cable lay and repair operations, but also military operations (e.g., mine countermeasures). The number of vessels with DPS has increased in the last decade. This is mainly due to increased oil and gas exploration at sea, as well as offshore operations, such as drilling, diving support, wind mill park maintenance, and anchor handling.

There is a long history of literature showing that operators do not function well as backup of automated systems (see, for example, Hancock et al., 2013; Onnasch et al., 2013; Wickens et al., 2015). Often, operators need more time to intervene under automated than under manual control. Operators need to first recover awareness of the state of the automated system before taking back control (see Metzger \& Parasuraman, 2005, p. 37). When an operator needs to regain control over a large oil platform after the initiation of a drive-off incident to prevent an imminent collision with another platform, this extra time is often not available. These types of problems related to changes in task demands stemming from automation have been summarized as the out-of-the-loop (OOTL) performance problem: the disability of human operators to control an automated system when automation fails or malfunctions (Metzger \& Parasuraman, 2005; Moray, 1986; Wickens and Kessel, 1979; Wiener and Curry, 1980).

Overall semi-autonomous system performance is highly dependent on how operator and automation function as a team (cf. Christoffersen and Woods, 2002; De Visser and Parasuraman, 2011). Both components are highly interdependent. Between manual control and full automation, different levels of automation, or collaboration forms, can be distinguished. Well known classifications are made by Sheridan and Verplank (1978) and by Endsley and Kaber (1999), with different variations. A special form of human-automation collaboration are adaptive systems. Adaptive systems are systems in which the locus of control between human and automation varies over time (Rouse, 1988; Parasuraman et al., 1996). This implies that the responsibility for a specific subtask moves from the automation to the operator or vice versa. Adaptive automation is a subset of an adaptive system. The preposition 'adaptive' implies that the automation takes over tasks from the human operator when the need arises, for instance due to high operator workload (cf. Parasuraman, 2000). Management by exception, as noted by Dekker and Woods (1999), is, in a figurative way of speaking, the mirror image of adaptive automation, where instead of the automation, the human supervisor, or exception manager, takes over tasks from the failing automation. This form of supervisory control bears high resemblance to the situation in which the DP operator is monitoring the semi-autonomous system for boundary conditions.

Exception managers often perform different tasks concurrently. Because human attention is limited, attention needs to be shifted between tasks (Squire and Parasuraman, 2010). Shifts in attention may result in fluctuations in awareness of the state of the supervisory control system and the environment (e.g., the weather, ship state). Moreover, it is often difficult for human operators to notice changes to relevant parameters that are under supervision (cf. Simons \& Rensink; Simons and Levin, 1997). This implies that the operator may be unaware of an unfolding emergency situation that requires operator involvement. When the need for operator involvement on the supervisory tasks becomes apparent, the operator must reassess the environment and system state to recover situation and system awareness, a process which Gartenberg et al. (2013) have called Situation Awareness Recovery (SAR).

Despite the increasing usage of semi-autonomous systems, little research has addressed the need for better tools to help human supervisors to recover awareness, for instance following interruptions in multitasking or emergency situations. This lack of research is surprising, because incidents may lead to considerable costs, especially in the maritime domain (cf. Payne, 2001). These costs include, but are not limited to, (1) injuries and fatalities, (2) severe equipment damage or destruction, (3) major pollution, and (4) rig downtime with significant loss of revenue and contractual problems. Because the estimated arrival of first-generation fully autonomous DPS is not foreseen in the near future, we thus need to consider how human operators could be aided in their role as backup for handling exceptional situations (cf. Endsley, 2016).

In this paper we address the question how to speed up SAR. We deduced several design principles for support of supervisors of highly automated systems to help them cope with regaining situation and system awareness as quickly as possible in emergency situations. Based on these principles, we then designed an adaptive support concept which we tested in a DP simulation. The remainder of this paper is structured as follows. First, we describe relevant literature to provide a better understanding of what situation awareness recovery is, how it may be distinguished from other forms of interruption management, and how this process may be supported. Next, we detail the support concept and our expectations on how this concept would compensate for the degrading effect of loss of awareness typically associated with the out-of-theloop performance problem. Then, we outline the present research and present the findings of our studies. We conclude by summarizing the implications of our research.

\subsection{Deciding on how to support Dynamic Positioning operators}

During stationary operations, the main goal of the DP operator is to maintain the platform on a predefined position. During this task the operator is supported by a DPS. A DPS is a highly autonomous system. Based on predefined settings they are able to keep the platform in position. Position sensors like Differential Global Positioning System (DGPS) sensors and gyro sensors give information about the platform's actual position. Wind sensors and information about current give input about the forces that work upon the platform. By using the right thruster power the platform is kept in place. A control system calculates the required power and position of the thrusters.

However, the system is not infallible. Sensors may not work perfectly, and thrusters and power supply can be suboptimal. Because of incorrect position information, or insufficient thrust the system may not be able to maintain position and the platform will drift off. The DP operator must recognise these failures in time and take appropriate actions to prevent position loss and accidents.

As long as the system works properly, DP operators do not have much work to do. However, the operator has to be constantly alert to detect failures on time and take immediate action. During interviews we asked two DP operators with extensive experience on Floating Production Storage and Offloading (FPSO) vessels about the cognitive strategies they use to detect these impending failures. One prominent strategy is monitoring for changes in key information presented through the DP interface. Changes in sensor values may indicate that something is wrong. For example, changes in thruster power values can be a sign that thrusters do not work properly. Changes on the state of the automation and the system it is controlling play an important role in the assessment of possible operational threats, so that the operator can take the right 
measures and prevent position loss.

The DPS supports the operator in this process by showing alerts and alarms when key values exceed operator settings. Still, the operator has to monitor these values constantly to detect deviations in time, preferably before the sounding of the alarm, a skill that people do not excel at as vigilance is reduced over even short periods (Hancock, 2013). Moreover, people are often 'blind' to changes to attended visual scenes, even for large changes, and especially when attention is limited or the change signal is masked (Simons and Levin, 1997; Simons and Rensink, 2005). People have also been found to greatly overestimate their ability to detect changes in their environment (Levin et al., 2000). Hence, people are often 'blind' to change blindness. Support that helps the human operator to detect significant changes in the state of the automation and the system it is controlling could help to detect that a problem exists and speed up the process of arriving at a sufficient understanding of the problem to intervene effectively (Endsley, 2016).

\subsection{Situation Awareness Recovery (SAR)}

SAR is the process of restoring situation awareness (SA) after it has been reduced (Gartenberg et al., 2013. Gartenberg et al. (2013) describe this process with the example of a task where multiple objects, for example unmanned aerial vehicles, have to be monitored simultaneously. They state that when one object requires attention, the operator's attention will primarily be focussed on that one object, due to constraints in attentional supply, resulting in loss of attention for the remaining objects. In order to regain awareness on the other objects, the operator must switch attention back to these objects to recover task states and goals of interrupted procedures from memory. Further, the operator must detect any significant changes that occurred during the interruption, assess their impact and respond as necessary (St. John and Smallman, 2008).

Several models have been developed in the past that offer a framework for characterizing SAR. The Memory For Goals (MFG) model describes the way goals direct behaviour and how memory elements determine the level of activation of a goal (Altmann and Trafton, 2002). Altmann and Trafton (2002) state that in a monitoring task each of the elements that have to be monitored make up a sub-goal. It is further argued that when one goal is active, i.e. directing behaviour, the other goals are suspended. This means that during an interruption another task directs behaviour suspending the primary task. A longer suspension of a goal results in a further reduction of the level of activation and a more substantial loss of SA for that task.

Another model characterizing SAR is the four-stage framework for maintaining and recovering situation awareness by St. John and Smallman (2008). The stages are: real-time change detection, preinterrupt preparation, post-interrupt reorientation, and post-hoc change detection. The first stage occurs pre-interruption and entails monitoring for changes as to not lose SA. Pre-interrupt preparation describes the time given to an operator to prepare for an upcoming interruption. It is thought that the shorter the preinterruption preparation time is, the more severe the SA loss will be. Post-interrupt reorientation occurs after an interruption and entails having to recall the previous goal and task state from memory or through external cues. This process is influenced by the duration of the interruption and the changeability of the situation. The final stage involves having to detect and comprehend changes that occurred during the interruption.

Squire and Parasuraman (2010) state that the resumption lag varies as a result of Level Of Automation (LOA). The resumption lag is longer during operations involving high LOA as compared to low LOA. During operations involving high LOA the operator is less involved in details about his environment as those are usually managed by the automatic system. This increases the difficulty of comparing a previous situation to the current situation after an interruption.

When looking at DP, the operators have to work in a highly automated environment, are frequently interrupted, sometimes for long periods of time during which situations could change rapidly, setting the stage for long resumption lags should manual control be required. Based on the MFG model and the four-stage framework for maintaining and recovering situation awareness, as discussed above, we propose that automated change detection, where an aid automatically detects and shows changes in the operational environment, can support the operator, especially in situations where the operator has low SA when overseeing automation and that require getting the operator back in the loop as fast as possible (cf. St. John and Smallman, 2008).

\subsection{The present research}

The goal of the present research is to test the utility of change detection support on operator monitoring in a simulated DP environment. For this purpose change detection of relevant DPS parameter values was automated. An experiment was designed to investigate whether automation of change detection enables operators with low awareness of the system state and the environment to quickly recover situation awareness in emergency takeover situations. Participants performed the simulated DP task with or without change detection support. The aid detects significant changes (decreases as well as increases) in input values and notifies the operator of these changes.

We manipulated whether or not there is a prolonged interruption during the task. According to the MFG theory, an interruption would lead to significant drops in situation awareness on the interrupted task and will induce situation awareness recovery after the interruption (cf. Gartenberg et al., 2013). Hence, we expect a worsening of performance in conditions with interruption as compared to conditions without interruption. Moreover, we expect that the aid would be especially beneficial during these situations of situation awareness recovery following an interruption, but not so much under continuous working conditions. Hence, in continuous working conditions, where operators are able to allot their full uninterrupted attention to the monitoring task for the short duration of the task, situation awareness is expected to be at high levels, and there is a low risk for 'change blindness'. An aid would have limited added value under such conditions, or, might even clutter the interface, making key information on the display more difficult to find and perceive (cf. Wolfe, 1998; Yeh et al., 2003).

To summarize, we hypothesize that interruptions would cause reductions in situation awareness. Further, we hypothesize that change detection automation would help in the process of recovering situation awareness after it has been reduced. More specifically, we hypothesize that performance and situation awareness will be enhanced with change detection automation, whereas overall mental workload will be reduced, but only in conditions when situation awareness has been reduced.

\section{Method}

\subsection{Participants}

A total of twenty-three DP-naive participants took part in this study. Student participants were recruited due to very limited availability of experienced DP operators. One participant was excluded after training due to a failure to reach the criterion to be allowed to take part in the experiment. The data reported here is based on the remaining twenty-two participants (12 male, 10 
female). Their age ranged from 18 to 28 years $(M=22.1 ; S D=2.4)$. It should be noted that in real operations, age range of operators is more widespread, with higher average age, and that predominantly males are fulfilling DP positions. Participants were paid $€ 35$ for participation and were promised another $€ 40$ for the best performing and $€ 20$ for the second best performing participant on the DP monitoring task to enhance motivation. Each participant had normal hearing and normal or corrected to normal vision.

\subsection{Materials: DP simulation environment}

We developed an in-house simulation capability, the DP simulation. The DP simulation was designed to isolate the relevant cognitive requirements associated with a single operator controlling maritime vessels operating on DP, while providing for a degree of experimental control. The design of the simulation was based on real DP systems and actual sensor information. The interface of the simulation is depicted in Fig. 1. Nine numeric parameters had to be monitored during twelve scenarios and ten training scenarios. The parameters were: Wind angle (degrees), Azimuth angle (angle of the Azimuth thruster in degrees), Azimuth thruster (\% power), Bow thruster (\% power), Wind strength $(\mathrm{m} / \mathrm{s})$, Setpoint error (distance from setpoint in meters), Wave height $(\mathrm{m})$, Roll angle (absolute degrees), and wave period (seconds/wave). These values were not fixed, but fluctuated around a pre-set value with a frequency of approximately $60 \mathrm{~Hz}$ and an amplitude of around $2 \%$ of the pre-set value.

Scenarios were based on a description of precursors of DP station keeping incidents as described by IMCA's Analysis of Station Keeping Incident Data over the period 1994-2003 (IMCA, 2006). Two DP operators with extensive experience on Floating Production Storage and Offloading (FPSO) vessels validated the scenario's. These were the same operators that we interviewed during the design stage of the experiment. Their feedback was used to further improve the realism of the scenario's. Scenarios included sudden wind increase, thruster failure, drifting, wave height increase, change of wind angle and various combinations.

\subsection{Task}

The task required participants to monitor the parameters during a monitoring interval for surpassing threshold values, strong linear increases or decreases, and unexpected halting of parameters. Some of these values were interdependent as well. During the training phase, participants learned information about these extreme situations.

In the middle of each scenario, a maximum of 6 parameters could begin to increase or decrease at different speeds. Or a parameter could halt at the current value, representing problems with displays or sensors, such as taut wires or GPS. The task of the participants was to respond to a loud auditory alarm signal at the end of the monitoring interval indicating that an action was required by them. Participants then had to respond as quickly as possible. A response was given in this action interval by mouse clicking on one of six action buttons, increasing in extremity of the situation: (1) 'Ok' (indicating that no action was required), (2) 'Monitor', (3) 'Caution', (4) 'Partial Takeover', (5) 'Full takeover', and (6) 'Detach' (see also Fig. 1).

According to the information that was provided about the thresholds (Table 1) and accompanying decision rules (Table 2), participants had to decide which action was correct. All information was trained to criterion during training and made available during the experiment, to relieve working memory load.

\subsection{Experimental design and independent variables}

We tested our hypotheses in a repeated measures factorial design, meaning that each participant received all treatments. Within-subjects factors were support (Yes, No) and interruption (Yes, No). The order of treatments was counterbalanced across subjects.

In the support conditions, participants were supported in the task with, what we have called, an Automated Change Recognition (ACR) system. The ACR system detected significant changes (decreases as well as increases) in input values, thereby off-loading participants of the responsibility of keeping track of changes, but not of the responsibility of using the sensor information to evaluate whether a response would be required. Hence, the automation did not simply take over the monitoring task from participants but supported the overall goal of making an accurate assessment of the situation (cf. Parasuraman et al., 2009). The support signalled significant changes and directions of these changes (up or down) to participants by depicting arrows on the monitoring screen next to the relevant values (see Fig. 2). The arrow appeared when a variable

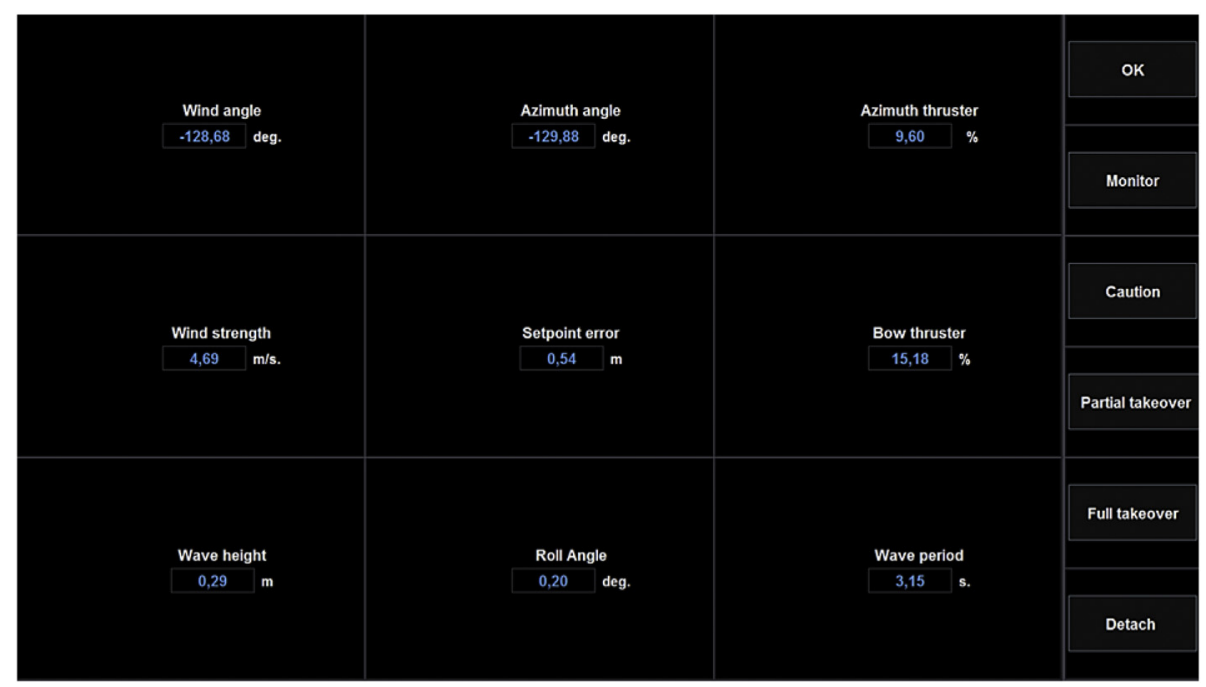

Fig. 1. The interface of the simulation at the start of a scenario. 
Table 1

Summary of variable attributes, thresholds, and how to determine when abnormalities arise.

\begin{tabular}{|c|c|c|c|c|}
\hline Variable & Min Threshold & Max Threshold & Delta & Delta an abnormality when: \\
\hline Wind angle & - & - & $20^{\circ}$ & Changed $( \pm)$ \\
\hline Azimuth angle & - & - & $20^{\circ}$ & Different from the wind angle \\
\hline Azimuth thruster & $5 \%$ & $95 \%$ & $15 \%$ & Decreased/frozen when Setpoint error increased \\
\hline Wind strength & $0 \mathrm{~m} / \mathrm{s}$ & $15 \mathrm{~m} / \mathrm{s}$ & $3 \mathrm{~m} / \mathrm{s}$ & Increased \\
\hline Setpoint Error & $-10 \mathrm{~m}$ & $10 \mathrm{~m}$ & $2 \mathrm{~m}$ & Increased or decreased from 0 \\
\hline Bow thruster & $5 \%$ & $95 \%$ & $15 \%$ & Decreased/frozen when Setpoint error increased \\
\hline Wave height & $0 \mathrm{~m}$ & $8 \mathrm{~m}$ & $2 \mathrm{~m}$ & Increased \\
\hline Roll Angle & $0 \mathrm{gr}$ & $20 \mathrm{gr}$ & $5^{\circ}$ & Increased \\
\hline Wave period & - & - & $5 \mathrm{~s}$ & Changed $( \pm)$ \\
\hline
\end{tabular}

Table 2

Converting detected sensor values and abnormalities into responses.

\begin{tabular}{lll}
\hline$\#$ & Response & Decision rule \\
\hline 1 & OK & No abnormalities or exceeded thresholds \\
2 & Monitor & 1 or 2 abnormalities \\
3 & Caution & 1 threshold exceeded or 3 abnormalities \\
4 & Partial takeover & 1 threshold exceeded and abnormalities \\
5 & Full takeover & 2 thresholds exceeded \\
6 & Detach & 2 thresholds exceeded and abnormalities \\
\hline
\end{tabular}

had increased or decreased during the past $5 \mathrm{~s}$, exceeding a predefined threshold. The arrow remained visible after it appeared, giving information that a value had been increasing but also remained on the screen if the variable was stable again. Hence, the arrow depicted whether variables had changed significantly over a fixed time interval. Participants were told beforehand that the change detection tool might be switched on during the scenario by the experimenter. As mentioned, the support system helped with perceiving significant changes, but participants still had to decide whether these changes were abnormalities or exceeding relevant thresholds, requiring imminent operator involvement.

In the interruption conditions, planned interruptions took place. For this purpose, each scenario was parsed in two discrete phases. An interruption took place in the second phase of the scenario, allowing participants some time prior to this interruption to acquire situational awareness. The interruption lasted till the end of the scenario. Hence, support was also turned on, depending on condition, at the beginning of the second phase of the scenario. During the interruption, participants were required to perform a filler task for approximately two minutes, the duration of the monitoring interval of the second phase of the scenario. This time period was based on the results of Monk et al. (2008), who found that short interruptions cause sufficient interference effects while still being practical in an experimental setting. At the sound of the alarm, a short but loud pulse, participants were instructed to immediately shift attention back to the primary DP monitoring task, assess the situation, and select the correct response as quickly as possible, thereby simulating emergency alarm handling. To make sure that the alarm was notable to all participants, and would not be obscured by ambient noise, the experiment was held in a silent room with sound absorbing walls and ceiling. The Visual Elevator Task (VET) was used as filler task. This is a subtask of the Test of Everyday Attention (TEA) developed by Robertson et al. (1996). The VET loads on cognitive functions such as memory and attentional switching.

\subsection{Procedure}

Upon arrival participants received general instructions, completed an informed consent form, and answered some demographic questions. Next, the training phase started. Participants were given general information about the work of a DP operator and what variables could have an influence on the state of the ship. Participants were told to imagine that they were a DP operator on a ship, and had to monitor the DP automation for failures.

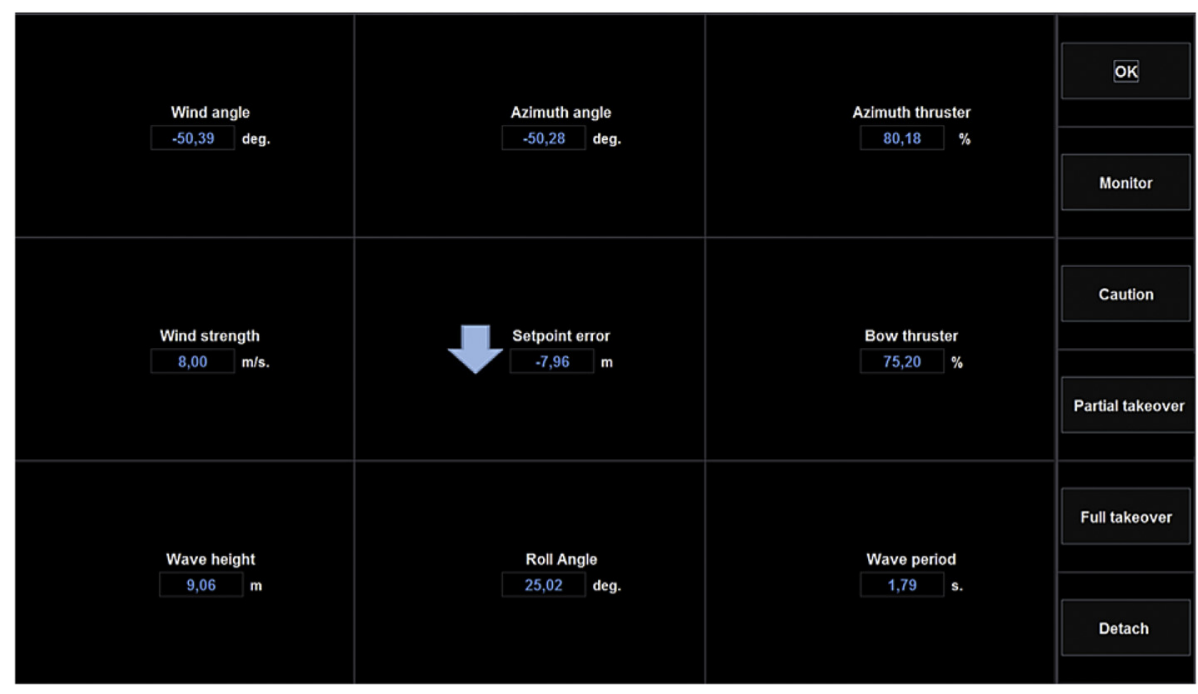

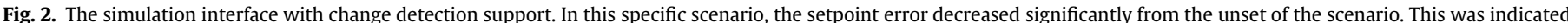

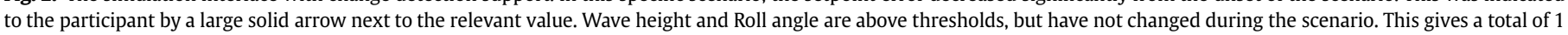
abnormality and 2 times a surpassing of thresholds. The correct decision would therefore be: Detach ( 2 thresholds exceeded + abnormalities). 
Participants were then instructed on rules describing which action is best under the given circumstances. After this instruction, the participants were trained on a maximum of ten practice scenarios divided over two blocks. The experimenter was present during these training trials, gave participants feedback on their performance, and evaluated at the end of the second training block whether participants reached the criterion of four of five correct responses to be allowed to take part in the experiment.

After a short break participants began with the first scenario. The experimenter was not present during task performance but observed the participants from behind a window in an adjacent room with sound absorbing walls and ceiling. The experimental setup is depicted in Fig. 3. The experiment consisted of 12 scenarios, 3 for each experimental condition. Each scenario lasted approximately $5 \mathrm{~min}$, depending on the response speed of the participants in the action interval. The order of presentation of scenarios was counterbalanced across participants. At the end of each completed scenario, after a response was given by the participants, they filled in questionnaires on situation awareness and workload.

\subsection{Dependent variables}

\subsubsection{Task performance: number of correct responses}

Participants were required at the end of the scenario to evaluate the best action to take given the current situation, yielding a total of 12 responses per participant, 3 for each condition. Task performance was determined by the number of correct responses, with 3 being the maximum for each condition, and 0 being the minimum.

\subsubsection{Task performance: reaction time for correct responses}

The reaction time was determined by the time it took participants in seconds to decide on the best action to take from the onset of the alarm, indicating the start of the action interval. Recall that the reaction time is hypothesized to be dependent on the interruption condition. After an interruption, participants need to refresh their memory. Hence, more time is needed to recover SA and take decisions accordingly. Because participants were instructed to perform at their best, and performance was measured be the number of correct responses, it is reasonable to assume that if an erroneous response was given, the monitoring role was not carried out 'normally' and, hence, could be regarded as contamination of the data (Sternberg, 2010). Therefore, only the response time for correct responses was used for further analysis.

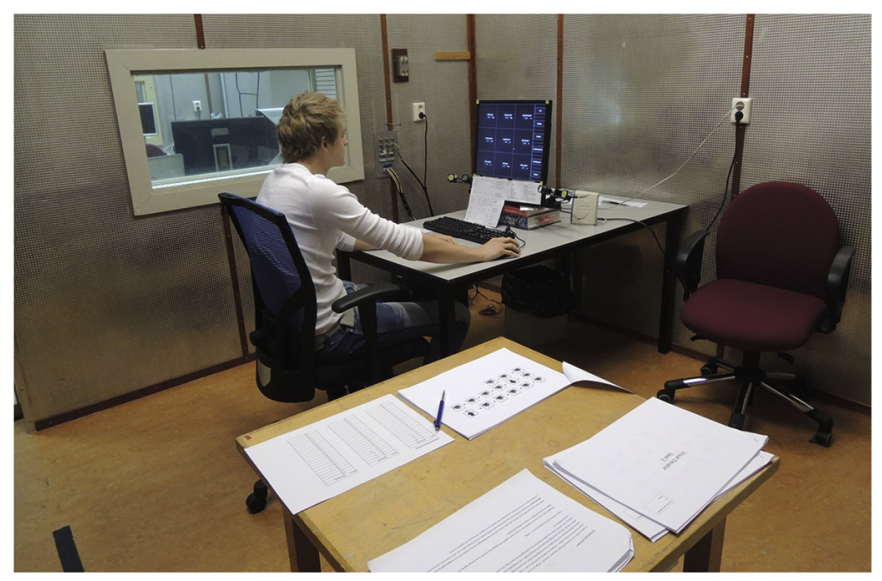

Fig. 3. Experimental setup (staged).

\subsubsection{Mental workload}

To evaluate mental workload, the Rating Scale Mental Effort (RSME) was administered directly after completion of each scenario. O'Donnell and Eggemeier (1986) define mental workload as the ratio between the task demands and the capacity of the operator working on the task. According to their definition, mental workload is high when the difference between task demands and capacity is small. The RSME is a one-dimensional scale with ratings between 0 and 150 (Zijlstra, 1993). Ratings of invested effort are indicated by putting a mark on a continuous line which runs from 0 to $150 \mathrm{~mm}$ with indications on every $10 \mathrm{~mm}$. Along the line, at several anchor points, statements related to invested effort are given (e.g., 12 corresponds to not effortful, 58 to rather effortful, and 113 to extremely effortful). A mental workload score is determined by measuring the distance from the origin to the mark in $\mathrm{mm}$. Previous research has shown that the RSME is good in providing a global rating of workload, while being easy and quick to administer (Hendy et al., 1993; Pickup et al., 2005; Van der Kleij, 2007; Veltman and Gaillard, 1993; Wierwille and Eggemeier, 1993).

\subsubsection{Situation awareness}

To measure situation awareness, the Situation Awareness Rating Technique (SART) was used (Taylor, 1990). The SART is a simplistic post-trial subjective rating technique. We chose to use the SART because it is quick and easy to use, requires little training, and, more importantly, is non-intrusive to task performance. Hence, the SART causes no interruption of the natural flow of the task, as opposed to some of the other techniques using simulation "freezes". This was important to us because we did not want to cause any unintended interruptions during task performance. Although some problems with gathering subjective SA data post-trial have been reported in literature, including memory degradation and poor recall, there are just as many studies indicating that the SART is an accurate and precise technique for measuring SA (see Salmon et al., 2009; and Salmon et al., 2006 for reviews).

The SART measures three separate constructs: Attentional Demand (D), Attentional Supply (S) and Attentional Understanding (U). The three component scores were determined with the 10-D SART questionnaire (Kennedy \& Durbin, 2005), consisting of 10 statements. All items were measured on seven-point Likert scales, in which a score of 1 corresponds to the most negative response to a statement, and a score of 7 corresponds to the most positive response to a statement. Three statements were used to assess demand, 4 for supply, and 3 statements for understanding. For the experiment, the questions were translated into Dutch and adapted to our setting. To calculate overall situation awareness, the mean scores per component were converted with formula (1) (cf. Satuf et al., 2016):

$S A=U-(D-S)=U+S-D$

With $S A$ being the Situation awareness score, $U$ the summed understanding, $D$ the summed demand and $S$ the summed supply. Hence, the minimum value of the scale was -14 . The maximum value was 46 .

Feature scaling was used to bring all values into a more comprehensible $0-100 \%$ range. The overall SA scores of the participants were normalized according to formula (2):

$\mathrm{Vn}=(\mathrm{v}-\mathrm{vmin} / \mathrm{vmax}-\mathrm{vmin}) * 100 \%$

Whereas $V n$ is the SA value in percentages, $v$ is the true SA value, $v m i n$ is the minimum value of the scale and vmax is the maximum value of the scale (see also Satuf et al., 2016). 


\section{Results}

Two-way repeated-measures Analysis of Variance (ANOVA) tests were conducted at the $p<.05$ level to determine the effects of our manipulations on our dependent variables. Because our a priori predictions were directional, ad-hoc differences between conditions were assessed by 1 -tailed paired samples $t$-tests, with a significance level of $p<.05$.

\subsection{Task performance: number of correct responses}

We found no significant main effect for interruption on the number of correct responses, $F(1,21)=1.12, p=.30$, partial $\eta^{2}=.05$. Moreover, the analysis revealed no significant main effect of support on the number of correct responses, $F(1,21)=.63, p=.44$, partial $\eta^{2}=.03$. The major effect of interest is whether there was a significant Interruption $x$ Support type cross-over interaction for the Number of correct responses. This was indeed the case, $F(1,21)=9.02, p<.01$, partial $\eta^{2}=.30$ (see Fig. 4 ).

Post hoc comparisons revealed that participants without support performed significantly worse when interrupted $(\mathrm{M}=.58 ; \mathrm{SD}=.32)$ than when working continuously $(\mathrm{M}=.80$; $\mathrm{SD}=.27), t(21)=3.07, p<.01$. Second, when no interruption was present, no significant difference was found between the not supported participants $(\mathrm{M}=.80 ; \mathrm{SD}=.27)$ and the supported participants $(\mathrm{M}=.70 ; \mathrm{SD}=.31), t(21)=1.23, p=.231$, meaning that under non-interrupted working conditions, support had no benefit to our participants. However, when an interruption was present, results show that participants performed significantly better with support $(M=.78 ; S D=.23)$ than without support $(\mathrm{M}=.58 ; \mathrm{SD}=.32), t(21)=-3.31, p<.001$. Fourth, an independent $t$-test was also used to determine whether support would allow interrupted participants to perform at the same performance levels as unsupported participants. Indeed, the test revealed no differences on task performance between interrupted participants with support $(\mathrm{M}=.78 ; \mathrm{SD}=.23)$ and participants working continuously without support, $\quad(\mathrm{M}=.80$; $\mathrm{SD}=.27), t(21)=0.24, p=. \mathrm{ns}$.

Taken together, these results suggest that interruptions negatively affect task performance. Our results also suggest that providing accurate monitoring support, i.e. on detecting significant changes in sensor values, to participants, has a positive effect on performance. But this result holds only in conditions where participants were interrupted, and, hence, had to allocate attention to a filler tasks.

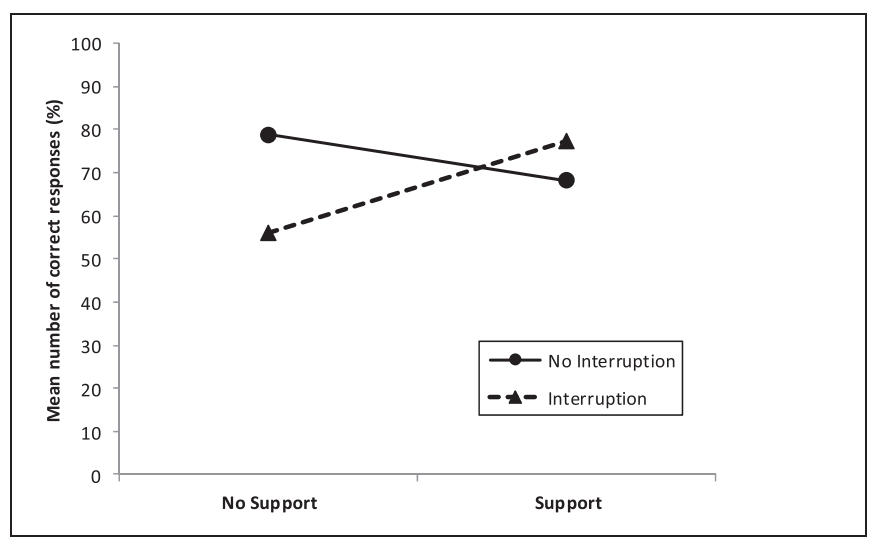

Fig. 4. Mean number of correct responses for supported and non-supported participants in interrupted and continuous task conditions.

\subsection{Task performance: reaction time for correct responses}

When looking at the response time for correct responses, analysis showed a significant main effect of interruption, $F(1,21)=80.06, p<.001$, partial $\eta^{2}=.79$. Participants responded significantly slower in the interruption conditions $(M=25.13$ s; $\mathrm{SD}=1.19 \mathrm{~s})$ than in the continuous task conditions $(\mathrm{M}=14.74 \mathrm{~s}$; $\mathrm{SD}=1.38 \mathrm{~s}$ ). This finding is consistent with other research that has shown increased resumption lags after interruptions (e.g., Gartenberg et al., 2013; Monk et al., 2004; Trafton et al., 2003). No main effect of support condition was found on response time, $F(1,21)=1.25, \mathrm{p}=$.ns, partial $\eta^{2}=.06$. Further, no significant interaction was found, $F(1,21)=2.66, p=$. ns, partial $\eta^{2}=.11$.

For purposes of hypothesis testing, we conducted two additional tests. First, we investigated more specifically whether support would help participants to respond more quickly after interruptions. Indeed, a significant difference was found between both interruption conditions, with and without support, $\mathrm{t}(21)=1.79, p=<.05$. Participants with support $(\mathrm{M}=23.88 \mathrm{~s}$; $\mathrm{SD}=6.48 \mathrm{~s}$ ) responded faster than participants without support $(\mathrm{M}=26.39 \mathrm{~s} ; \mathrm{SD}=6.56 \mathrm{~s})$. Second, we wanted to know whether support would help interrupted participants to approach response speeds of participants in the unsupported, non-interrupted condition. This was not found to be the case. The second $t$-test revealed significant differences on reaction time between interrupted participants with support $(\mathrm{M}=23.88 \mathrm{~s} ; \mathrm{SD}=6.48 \mathrm{~s})$ and participants working continuously without support, $(M=14.64 \mathrm{~s}$; $S D=7.52 \mathrm{~s})$, $t(21)=-4.12, p<.001$. The interrupted participants with support were significantly slower in responding to the alarm signal than unsupported, non-interrupted participants. So, although support helped participants after interruptions to take actions more quickly, the response time, however, did not approach the response times of participants in the non-interrupted conditions.

\subsection{Mental workload}

A significant main effect of interruption was found on mental workload, $F(1,21)=39.55, p<.001$, partial $\eta^{2}=.65$. Participants in the interruption condition experienced more mental workload $(\mathrm{M}=72.92 ; \mathrm{SD}=3.88)$ than participants in the non-interruption condition $(M=51.15 ; S D=3.76)$. No significant main effect for support was found on mental workload, $F(1,21)=2.05, p=$.ns, partial $\eta^{2}=.09$. Interestingly, an interaction was found between interruption and support, $F(1,21)=6.65, p<.05$, partial $\eta^{2}=.24$ (see Fig. 5).

Again, we ran several paired samples $t$-tests to better understand the nature of the interaction. First, participants without support experienced significantly more mental workload when interrupted $(M=78.55 ; S D=22.90)$ than when not interrupted $(M=49.60 ; S D=20.15), t(21)=-6.32, p<.001$. Similar results were found when participants were supported. Mental workload was significantly higher in the interruption condition $(M=67.29$; $\mathrm{SD}=17.58)$ as compared to the non-interrupted condition $(\mathrm{M}=52.69 ; \mathrm{SD}=19.87), t(21)=-3.40, p<.01$. Third, when we look more closely at participants that were interrupted, we see that participants that were supported reported significantly lower levels of mental workload $(\mathrm{M}=67.29 ; \mathrm{SD}=17.58)$, than non-supported participants $(\mathrm{M}=78.55 ; \mathrm{SD}=22.90), t(21)=2.85, p=.01$. However, mental workload was still significantly higher than in the unsupported, non-interrupted condition ( $\mathrm{M}=49.60 ; \mathrm{SD}=20.15), t$ $(21)=-4.73, p<.001$.

\subsection{Situation awareness}

No main effect of interruption was found on overall SA, 


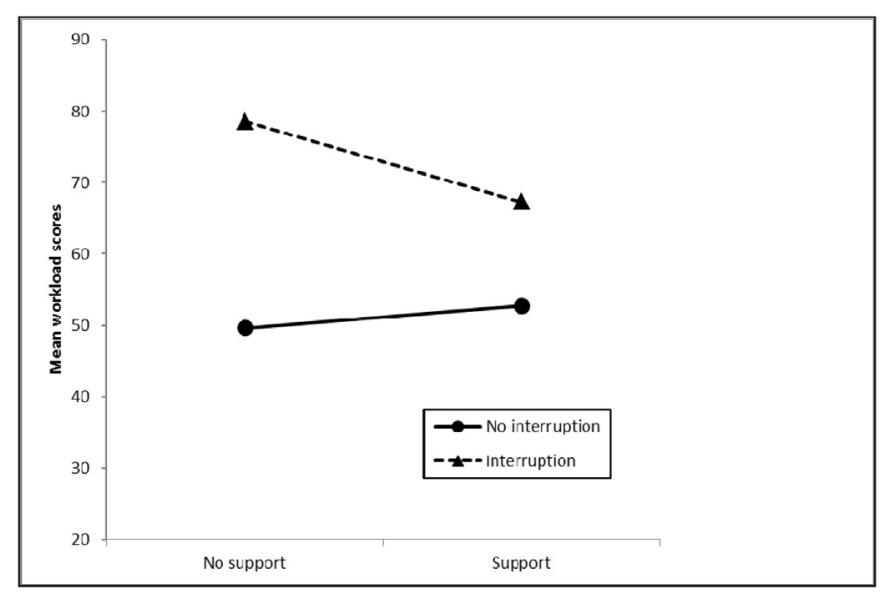

Fig. 5. Mental workload for supported and non-supported participants in interrupted and continuous task conditions.

$F(1,21)=2.17, p=. \mathrm{ns}$, partial $\eta^{2}=.09$. There was a significant main effect of support on SA, $F(1,21)=5.70, p<.05$, partial $\eta^{2}=.21$. Participants with support reported higher levels of SA $(M=64.24 \%$; $\mathrm{SD}=2.10 \%)$ than participants without support $(\mathrm{M}=61.41 \%$; $\mathrm{SD}=1.95 \%)$. Again, an Interruption $\mathrm{x}$ Support type cross-over interaction was found, $F(1,21)=15.18, p<.01$, partial $\eta^{2}=.43$ (see Fig. 6).

Paired samples $t$-tests confirmed our hypotheses. First, when participants were not supported, SA was significantly lower in the interruption condition ( $\mathrm{M}=58.54 \%$; $\mathrm{SD}=9.99 \%)$ than in the noninterruption condition ( $M=64.29 \% ; S D=10.09 \%), t(21)=3.42$, $p<.01$. Second, when no interruption was present, no differences in SA were found between participants without support $(M=64.29 \%$; $\mathrm{SD}=10.09 \%)$ and those with support $(\mathrm{M}=63.66 \%$; $\mathrm{SD}=10.34 \%)$, $t(21)=.625, p=$.ns. Third, in the interruption conditions, the participants with support reported a significantly higher level of SA $(\mathrm{M}=64.82 \% ; \mathrm{SD}=11.03 \%)$, than participants without support $(\mathrm{M}=58.54 \%$; $\mathrm{SD}=9.99 \%), t(21)=-4.22, p<.001$. Fourth, as expected, no differences in SA were found between participants that were not interrupted nor had support ( $M=64.29 \%$; $S=10.09 \%)$, and participants that were interrupted with support $(M=64.82 \%$; $\mathrm{SD}=11.03 \%), t(21)=-.27, p=. \mathrm{ns}$.

A more detailed look at the three SA components attentional demand, attentional supply and understanding showed similar

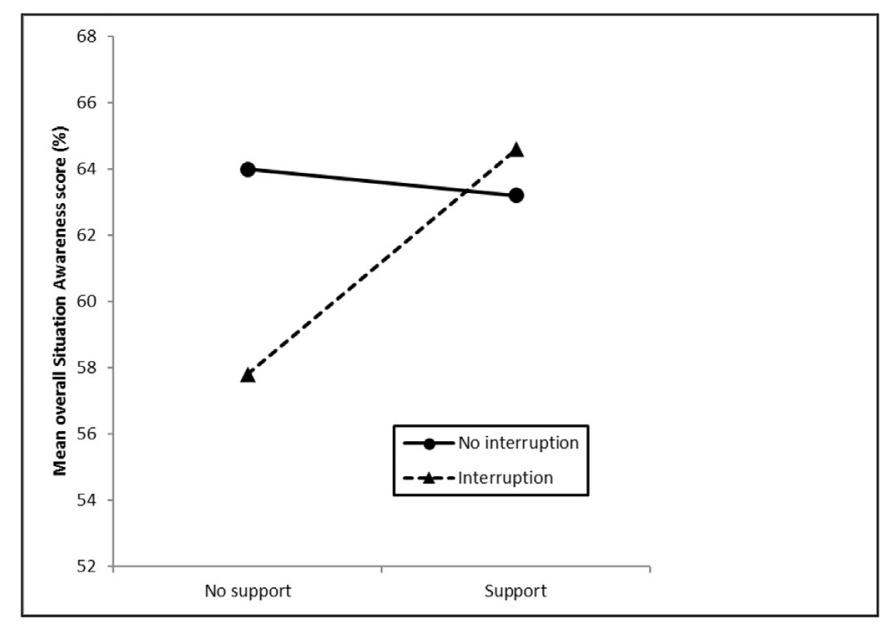

Fig. 6. Overall Situation Awareness in percentages for supported and non-supported participants in interrupted and continuous task conditions. results, except for attentional supply, as was to be expected. No differences between conditions were present for attentional supply, indicating that our manipulations had no effect on participants' resources of attention.

\section{Discussion}

Our primary goal was to investigate whether support of human change detection functions (i.e., the ability to detect relevant changes in the environment) would enable supervisory controllers to better resume a monitoring task after an interruption. A change detection aid was designed to enable controllers, when the need for involvement on a monitoring task becomes emergent, to reassess the automation and system state quickly. Hence, the change detection aid was predicted to help improve the supervisory controller's ability to quickly recover situation awareness in emergency take-over situations.

Our results show that change detection support helps in the process of recovering situation awareness. Interrupted participants performed more accurately and faster with the change detection aid than participants who were not supported. Moreover, we found that situation awareness was enhanced, whereas overall mental workload was reduced, as hypothesized.

Although change detection support helped interrupted participants to perform at better levels than their unsupported but also interrupted counterparts, it did not enable them to completely match performance levels of non-interrupted participants. On the one hand, in terms of accuracy of performance, support did enable interrupted participants to perform as accurately as noninterrupted participants. On the other hand, in terms of speed of performance, support could not compensate the longer reaction times caused by interruptions. Hence, even with support, there was still a resumption lag present. MFG theory predicts that after an interruption, supervisory controllers take longer to take action because of the need to reactivate decayed episodic memory chunks (Altmann and Trafton, 2002). Our support was designed to alleviate memory load and help controllers to detect post interrupt changes. Participants were liberated from the burden to remember relevant sensor values during interruptions. Why was our support then unable to reduce this lag completely?

St. John and Smallman's (2008) integrated framework for maintaining and recovering SA helped us to answer the question why the support did not completely eliminate the resumption lag. St. John and Smallman (2008) organized recovering SA after an interruption into two stages: (a) reorientation, and (b) change detection. Our support clearly supported the change detection stage, hence, explaining the reduction in response time for supported participants, but, given the results, not the post-interrupt reorientation stage. The reorientation stage "involves retrieving the primary task goal structure and primary task state prior to the interruption from either memory or the task environment-in other words, 'What was I doing?' "' (St. John and Smallman, 2008, p. 124). Our support was not designed to communicate this information to participants. Thus, our results are in support of St. John and Smallman's integrated framework, demonstrating that recovering SA consists of at least two stages, including post hoc change detection.

Interestingly, support had no added value under noninterrupted working conditions. That is, when participants were not interrupted, but still had to detect changes in real-time, the change detection automation had no added value whatsoever. This could only mean that, in non-interrupted working conditions, when participants were able to allot their full attention to the monitoring task for the short duration of the task, there was no fundamental loss of situation awareness. An aid for quickly 
recovering SA obviously has no added value in situations of high SA when overseeing automation.

Another remarkable finding were the negative effects of support in the non-interrupted conditions (cf. Bainbridge, 1983). Debriefing comments revealed that participants were annoyed by the support in the non-interrupted conditions. Participants complained that the support meant more information to attend to, and distracted them from performing the primary monitoring task, with as a consequence increasing levels of mental workload. This result confirms the findings of others on consequences of automation on human performance (cf. Dekker and Woods, 2002: The substitution myth; Wilson and Russell, 2007, p. 1016).

Our results have practical implications as well. The results show that change detection support has value in operational environments. Change detection support helps in situations where the operator has impaired SA and that require getting the operator back in the loop as fast as possible. However, our results also clearly show that adding support to an already highly automated DPS should be done with caution. Although support helped operators with low SA due to an interruption to recover quickly, performance in emergency situations was still below that of non-interrupted supervisory controllers. Moreover, support could also bring about unwanted or unanticipated effects (cf. Bainbridge, 1983). In our experiment we saw that the support was not beneficial to the operator in all conditions. Under continuous, non-interrupted conditions, the support resulted in higher operator workload, raising questions whether supervisory controllers should be supported continuously or only when it is required (cf. De Visser and Parasuraman, 2011).

Future research should investigate whether more adaptive forms of support could alleviate some of the negative effects of the support for the operator. The present study investigated the effects of static, leave on, support on performance of supervisory controllers of highly automated systems during emergency situations requiring operator involvement. Meaning that support was turned on, no matter the performance of the participants. For interrupted participants this turned out to be beneficial. For the uninterrupted participants, this was not the case. Hence, there were no performance nor model based justifications for the support, making it rather inflexible. Adaptive support has been proposed as a solution to the problems associated with inflexible automation (De Visser and Parasuraman, 2011; Inagaki, 2003; Kaber and Endsley, 2004; Parasuraman, 2000; Scerbo, 2001). Adaptive support is support "that is not fixed at the design stage but varies appropriately with context in the operational environment" (De Visser and Parasuraman, 2011, p. 211).

It would be interesting to see whether SAR processes could be used as a trigger for adaptive automation. A trigger could be the detection of SAR processes or the drop of an operator's level of SA below a certain threshold. SAR is characterised by "shorter eye fixation durations, increased number of objects scanned, and a greater likelihood of refixating on objects that were previously looked at" (Gartenberg et al., 2013, p. 710). Gartenberg and colleagues conclude that SAR consists of increased scanning in order to compensate for decay. Previously viewed cues act as associative primes. These cues are able to reactivate memory traces of goals and plans. Hence, the inclusion of real time analysis of patterns of eye movements that could serve as a trigger for support, would, in our opinion, be an interesting add-on to the current research.

According to Multiple Resource Theory (MRT) cognitive resources are limited (Wickens, 1984). A supply and demand problem occurs when humans performs two or more tasks that require a single resource. The attentional task participants performed in this experiment during interruptions loads highly on memory functions. As mentioned, during these interruptions, participants must also remember previous goal and task state to be able upon return to the suspended task to detect any significant changes that occurred during the interruption. This process is influenced by the duration of the interruption and the changeability of the situation, but, as postulated by MRT, also by the work that is being performed during the interruption. Hence, given the fact that the filler task loads highly on similar cognitive functions as needed for successfully resuming the primary DP task after interruptions, it is likely that this specific filler task has amplified the negative effect of interruptions on performance. Future research should look into this matter more closely.

\section{Conclusions}

Our study showed that change detection support helps in recovering situation awareness: Participants performed better and faster, their mental workload was reduced, and their SA was increased. However, change detection support is no magic bullet: Interrupted participants with support were not as fast as noninterrupted participants. This result is in support of St. John and Smallman's 2008 four-stage framework for maintaining and recovering situation awareness, demonstrating that recovering SA consists of at least two stages, including post hoc change detection. Interestingly, interrupted participants with support also perceived higher levels of mental workload than non-supported participants. This last finding raises the question whether supervisory controllers should be supported continuously or only when it is required. Future research should investigate whether adaptive aiding could alleviate some of the negative effects of non-adaptive operator support in highly automated work settings.

\section{Acknowledgments}

Funding: The research reported here was supported by TNO's Early Research Program (ERP) on Human Enhancement.

The authors would like to thank Jouke Rypkema, Hans van den Broek, Guido te Brake, Jasper van der Waa, and Max Tijdeman for their assistance with this research effort. We are also grateful to the participants of the 2016 International symposium: Human Factors and Automation, that took place in The Netherlands on October 12 and 13 , for their valuable comments on our research presentation.

\section{References}

Altmann, E.M., Trafton, J.G., 2002. Memory for goals: an activation-based model. Cognit. Sci. 26 (1), 39-83.

Bainbridge, L., 1983. Ironies of automation. Automatica 19 (6), 775-779.

Christoffersen, K., Woods, D.D., 2002. How to make automated systems team players. Adv. Hum. Perform. Cognit. Eng. Res. 2, 1-12.

Dekker, S.W., Woods, D.D., 1999. To intervene or not to intervene: the dilemma of management by exception. Cognit. Technol. Work 1 (2), 86-96.

Dekker, S.W., Woods, D.D., 2002. MABA-MABA or abracadabra? Progress on human-automation co-ordination. Cognit. Technol. Work 4 (4), 240-244.

De Visser, E., Parasuraman, R., 2011. Adaptive aiding of human-robot teaming effects of imperfect automation on performance, trust, and workload. J. Cognit. Eng. Decis. Making 5 (2), 209-231.

Endsley, M.R., 2016. From here to autonomy: lessons learned from human-automation research. Hum. Factors: J Hum Factors Ergon. Soc. https:// doi.org/10.1177/0018720816681350.

Endsley, M.R., Kaber, D.B., 1999. Level of automation effects on performance, situation awareness and workload in a dynamic control task. Ergonomics 42, $462-492$.

Fossen, T.I., 1994. Guidance and Control of Ocean Vehicles. John Wiley \& Sons, Inc.

Fitts, P.M., 1951. Human Engineering for an Effective Air-navigation and Trafficcontrol System. Ohio State University Research Foundation, Columbus Ohio.

Gartenberg, D., Breslow, L., McCurry, J.M., Trafton, J.G., 2013. Situation awareness recovery. Hum. Factors: J Hum Factors Ergon. Soc. 56, 710-727. DOI: 0018720813506223.

Hancock, P., 2013. In search of vigilance: the problem of iatrogenically created psychological phenomena. Am. Psychol. 68, 97-109.

Hancock, P.A., Jagacinski, R.J., Parasuraman, R., Wickens, C.D., Wilson, G.F., 
Kaber, D.B., 2013. Human-automation interaction research past, present, and future. Ergon. Des.: Q. Hum. Factors Appl. 21 (2), 9-14.

Hendy, K., Hamilton, K., Landry, L., 1993. Measuring subjective workload: when is one scale better than many? Hum. Factors 35, 579-601. https://doi.org/10.1177/ 001872089303500401.

IMCA, 2006. Analysis of Station Keeping Incident Data 1994-2003. The International Marine Contractors Association. IMCA M-181.

Inagaki, T., 2003. Adaptive automation: sharing and trading of control. In: Hollnagel, E. (Ed.), Handbook of Cognitive Task Design. Lawrence Erlbaum, Mahwah, NJ, pp. 147-170.

Kaber, D.B., Endsley, M., 2004. The effects of level of automation and adaptive automation on human performance, situation awareness and workload in a dynamic control task. Theor. Issues Ergon. Sci. 5, 113-153.

Kennedy, J.S., Durbin, D.B., 2005. Human Factors Assessment of the UH-60M Crew Station During the Early User Demonstration Number 2 (EUD2) (No. ARL-MR0607). Army Research Lab, Aberdeen Proving Ground MD.

Levin, D.T., Momen, N., Drivdahl, S.B., Simons, D.J., 2000. Change blindness blindness: the metacognitive error of overestimating change-detection ability. Vis. Cognit. 7, 397-412.

Metzger, U., Parasuraman, R., 2005. Automation in future air traffic management: Effects of decision aid reliability on controller performance and mental workload. Hum. Factors 47 (1), 35-49.

Monk, C.A., Boehm-Davis, D.A., Mason, G., Trafton, J.G., 2004. Recovering from interruptions: implications for driver distraction research. Hum. Factors 46 (4), 650-663.

Monk, C.A., Trafton, J.G., Boehm-Davis, D.A., 2008. The effect of interruption duration and demand on resuming suspended goals. J. Exp. Psychol. Appl. 14, 299-313. https://doi.org/10.1037/a0014402.

Moray, N., 1986. Monitoring behavior and supervisory control. In: Baff, K. (Ed.), Handbook of Perception and Human Performance. Wiley, New York (pp. 4011-40/51).

O'Donnell, C.R., Eggemeier, F.T., 1986. Workload assessment methodology. In: Boff, K.R., Kaufman, L., Thomas, J.P. (Eds.), Handbook of Perception and Human Performance: Vol. II. Cognitive Processes and Performance. Wiley, New York (pp. 42.1-42.29).

Onnasch, L., Wickens, C.D., Li, H., Manzey, D., 2013. Human performance consequences of stages and levels of automation: an integrated meta-analysis. Hum. Factors: J Hum Factors Ergon. Soc. 56, 476-488. DOI: 0018720813501549.

Parasuraman, R., 2000. Designing automation for human use: empirical studies and quantitative models. Ergonomics 43, 931-951.

Parasuraman, R., Cosenzo, K.A., De Visser, E., 2009. Adaptive automation for human supervision of multiple uninhabited vehicles: effects on change detection, situation awareness, and mental workload. Mil. Psychol. 21 (2), 270.

Parasuraman, R., Mouloua, M., Molloy, R., 1996. Effects of adaptive task allocation on monitoring of automated systems. Hum. Factors: J Hum Factors Ergon. Soc. 38 (4), 665-679.

Payne, J.C. (Ed.), 2001. Communications - the Forgotten Factor. Dynamic Positioning Conference. September 18-19, 2001. Houston, Texas.

Pickup, L., Wilson, J.R., Norris, B.J., Mitchell, L., Morrisroe, G., 2005. The integrated workload scale (IWS): a new self-report tool to assess railway signaller workload. Appl. Ergon. 36, 681-693. https://doi.org/10.1016/j.apergo.2005.05.004.

Robertson, I.H., Ward, T., Ridgeway, V., Nimmo-Smith, I.A.N., 1996. The structure of normal human attention: the Test of everyday attention. J. Int. Neuropsychol. Soc. 2 (06), 525-534.

Rouse, W.B., 1988. Adaptive aiding for human/computer control. Hum. Factors 30, 431-438.

Rousseau, J., Crane, B., 2016. The human transition to autonomous vehicles. Retrieved from the Internet on August $3^{\text {rd }}$ 2016, from Artefact. https://www. artefactgroup.com/content/the-human-transition-to-autonomous-vehicles/.

Salmon, P., Stanton, N., Walker, G., Green, D., 2006. Situation awareness measurement: a review of applicability for C4i environments. Appl. Ergon. 37 (2), $225-238$.
Salmon, P.M., Stanton, N.A., Walker, G.H., Jenkins, D., Ladva, D., Rafferty, L., Young, M., 2009. Measuring Situation Awareness in complex systems: comparison of measures study. Int. J. Ind. Ergon. 39 (3), 490-500.

Satuf, E.N., Kaszkurewicz, E., Schirru, R., de Campos, M.C.M.M., 2016. Situation awareness measurement of an ecological interface designed to operator support during alarm floods. Int. J. Ind. Ergon. 53, 179-192.

Scerbo, M., 2001. Adaptive automation. In: Karwowski, W. (Ed.), International Encyclopedia of Ergonomics and Human Factors. Taylor \& Francis, London, UK, pp. 1077-1079.

Sheridan, T.B., Verplank, W.L., 1978. Human and Computer Control of Undersea Teleoperators. Massachusetts Inst of Tech Cambridge Man-Machine Systems Lab.

Simons, D.J., Levin, D.T., 1997. Change blindness. Trends Cogn. Sci. 1, 261-267.

Simons, D.J., Rensink, R.A., 2005. Change blindness: Part, present, and future. Trends Cogn. Sci. 9, 16-20.

Sternberg, S., 2010, March 20. Reaction-time experimentation. Proseminar in psychological methods. Retrieved from. http://www.psych.upenn.edu/ saul/rt. experimentation.pdf.

St John, M., Smallman, H.S., 2008. Staying up to speed: four design principles for maintaining and recovering situation awareness. J. Cognit. Eng. Decis. Making 2. $118-139$.

Squire, P.N., Parasuraman, R., 2010. Effects of automation and task load on task switching during human supervision of multiple semi-autonomous robots in a dynamic environment. Ergonomics 53, 951-961.

Taylor, R.M., 1990. Situational awareness rating technique (SART): the development of a tool for aircraft systems design. In: Situational Awareness in Aerospace Operations (Rep. No. AGARDCP-478). NATO-AGARD, Neuilly Sur Seine, France, pp. 341-346.

Trafton, J.G., Altmann, E.M., Brock, D.P., Mintz, F.E., 2003. Preparing to resume an interrupted task: Effects of prospective goal encoding and retrospective rehearsal. Int. J. Hum.-Comput. Stud. 58 (5), 583-603.

Van der Kleij, R., 2007. Overcoming Distance in Virtual Teams: Effects of Communication Media, Experience, and Time Pressure on Distributed Teamwork. Doctoral dissertation. University of Amsterdam, Amsterdam, The Netherlands.

Veltman, J.A., Gaillard, A.W.K., 1993, April. Measurement of pilot workload with subjective and physiological techniques. In: Paper Presented at the Royal Aeronautical Society on the Assessment of Workload and Aviation Safety.

Wilson, G.F., Russell, C.A., 2007. Performance enhancement in an uninhabited air vehicle task using psychophysiologically determined adaptive aiding. Hum. Factors: J Hum Factors Ergon. Soc. 49 (6), 1005-1018.

Wickens, C.D., 1984. Processing resources in attention. In: Parasuraman, R. Davies, D.R. (Eds.), Varieties of Attention. Academic Press, New York, pp. 63-102.

Wickens, C.D., Clegg, B.A., Vieane, A.Z., Sebok, A.L., 2015. Complacency and automation bias in the use of imperfect automation. Hum. Factors 57 (5), 728-739.

Wickens, C.D., Kessel, C., 1979. The effect of participatory mode and task workload on the detection of dynamic system failures. IEEE Trans. Syst. Man Cybern. 9, $24-34$.

Wiener, E.L., Curry, R.E., 1980. Flight-deck automation: promises and problems. Ergonomics 23 (10), 995-1011.

Wierwille, W.W., Eggemeier, F.T., 1993. Recommendations for mental workload measurement in a test and evaluation environment. Hum. Factors 35, 263-281.

Wolfe, J.M., 1998. Visual search. Attention 1, 13-73.

Woods, D.D., Cook, R.I., 2006. Incidents: markers of resilience or brittleness? In: Hollnagel, E., Woods, D.D., Leveson, N. (Eds.), Resilience Engineering: Concepts and Precepts. Ashgate, Aldershot, UK, pp. 69-76.

Yeh, M., Merlo, J.L., Wickens, C.D., Brandenburg, D.L., 2003. Head up versus head down: the costs of imprecision, unreliability, and visual clutter on cue effectiveness for display signaling. Human Fact. 45 (3), 390-407.

Zijlstra, F.R.H., 1993. Efficiency in Work Behaviour: a Design Approach for Modern Tools. Doctoral dissertation. Delft University of Technology, Delft, The Netherlands. 